\title{
An important role for peroxiredoxin II in survival of A549 lung cancer cells resistant to gefitinib
}

\author{
Taeho Kwon ${ }^{1,2}$, Jin Kyung Rho ${ }^{3}$, Jae Cheol Lee ${ }^{3}$, Young-Ho Park ${ }^{1}$, Hye-Jun Shin ${ }^{1}$, Sunwha Cho ${ }^{4}$ \\ Yong-Kook Kang ${ }^{4}$, Bo-Yeon Kim ${ }^{5}$, Do-Young Yoon ${ }^{2}$ and Dae-Yeul Yu ${ }^{1}$
}

Redox adaptation is an important concept that explains the mechanisms by which cancer cells survive under persistent endogenous oxidative stress and become resistant to certain anticancer agents. To investigate this concept, we determined the expression levels of peroxiredoxins (Prxs), antioxidant enzymes in drug-resistant non-small cell lung carcinoma cells. Prx II was remarkably increased only in A549/GR (gefitinib-resistant) cells compared with A549 cells, consistent with methylation/ demethylation. Prx II was highly methylated in the A549 cells but was demethylated in the A549/GR cells. The elevated expression of Prx II resulted in the downregulation of reactive oxygen species (ROS) and cell death and upregulation of cell cycle progression in the A549/GR cells. When Prx II mRNA in the A549/GR cells was knocked down, the levels of ROS and apoptosis were significantly recovered to the levels of the controls. In addition, signaling molecules involved in apoptosis were increased in the A549/GR-shPrx II cells. There was no difference in the expression of MAPK/ERK between the A549/GR cells and A549/GRshPrx II cells, but the phosphorylation of JNK was increased in the A549/GR cells and was markedly decreased in the A549/GRshPrx II cells. Colony number and tumor growth were significantly decreased in the A549/GR-shPrx II cells compared with the A549/GR cells. Our findings suggest that Prx II has an important role in cancer cell survival via the modulation of signaling molecules involved in apoptosis and the phosphorylation of JNK by the downregulation of ROS levels in A549/GR cells. Experimental \& Molecular Medicine (2015) 47, e165; doi:10.1038/emm.2015.24; published online 29 May 2015

\section{INTRODUCTION}

Lung cancer is one of the leading causes of cancer-related mortality worldwide, with a 5 -year survival rate of only $15 \%$ for non-small cell lung cancer (NSCLC). ${ }^{1}$ Despite dramatic advances in the treatment strategies, including radiotherapy and chemotherapy, the patient survival rate has not improved. ${ }^{2}$ Gefitinib (Iressa, 4-(3-chloro-4-fluoroanilino)-7-methoxy-6(3-morpholinopropoxy)-quinazoline) is orally active and exerts its anti-tumor activity through the inhibition of EGFR tyrosine kinase. It has been used for treating advanced NSCLC with good tolerability. However, its activity has not been significantly correlated with EGFR expression by tumor cells. ${ }^{3}$ Some reports have shown differences between gefitinib responders and nonresponders in the frequency of activating mutations in the EGFR gene, suggesting that these mutations might be predictive markers for sensitivity to gefitinib. ${ }^{4}$ Among them, the EGFR-TK somatic mutation has been the focus of many studies because of its involvement in the response to gefitinib and patient survival. Some prospective studies have recently confirmed its role in the responses to EGFR-TK inhibitors, although its impact on survival is still unclear. ${ }^{4}$ Recently, a gefitinib-resistant subline (A549/GR) derived from the parental A549 cell line has been established by repeated exposure to gefitinib. Compared with A549 cells, A549/GR cells are more resistant to gefitinib. A549/GR cells are spindle-shaped, lack polarity and exhibit increased pseudopodia formation and invasion and migratory abilities. ${ }^{5}$ Further, gefitinib inhibits tumor cell growth and induces the production of reactive oxygen species (ROS). ${ }^{6}$

ROS are involved in the initiation and progression of cancer. ${ }^{7}$ The peroxiredoxins (Prxs) family is induced by various oxidative stimuli and provides important protection from oxidative radical damage by ROS. ${ }^{8}$ Prx, which has a pivotal role in removing $\mathrm{H}_{2} \mathrm{O}_{2}$, is ubiquitously present in large

\footnotetext{
${ }^{1}$ Disease Model Research Laboratory, Aging Intervention Research Center, Korea Research Institute of Bioscience and Biotechnology (KRIBB), Daejeon, Korea; ${ }^{2}$ Department of Bioscience and Biotechnology, Bio/Molecular Informatics Center, Konkuk University, Seoul, Korea; ${ }^{3}$ Asan Institute for Life Sciences, Asan Medical Center, College of Medicine, University of Ulsan, Seoul, Korea; ${ }^{4}$ Development and Differentiation Research Center, Korea Research Institute of Bioscience and Biotechnology (KRIBB), Daejeon, Korea and ${ }^{5}$ World Class Institute, Korea Research Institute of Bioscience and Biotechnology (KRIBB), Chungbuk, Korea

Correspondence: Professor D-Y Yu, Disease Model Research Laboratory, Aging Intervention Research Center, Korea Research Institute of Bioscience and Biotechnology (KRIBB), 125 Gwahak-ro, Yuseong-gu, Daejeon 305-806, Korea.

E-mail: dyyu10@kribb.re.kr
}

Received 31 October 2014; revised 22 December 2014; accepted 19 January 2015 
amounts in every living organism, ranging from bacteria, yeast and fungi to higher mammalian cells. ${ }^{9}$ Although the exact roles of Prx in signal transduction, cellular proliferation, carcinogenesis and protein chaperoning in living cells are unclear, the oxidation and reduction of sulfhydryl residues on the Prx protein may act as a molecular on-off signal similar to protein phosphorylation and dephosphorylation, which serve as molecular switches of protein activation. ${ }^{10}$ Many studies have indicated that Prxs is aberrantly expressed in various types of cancers. ${ }^{11}$ The Prx family is highly expressed in human lung carcinoma, with differences between the various Prxs, tumor histopathologies and their potential effects on tumor progression. In particular, Prx I, II, IV and VI are highly expressed in lung carcinomas. ${ }^{12}$ Prx II expression has been significantly associated with tumor progression. ${ }^{13}$ However, the role and mechanism of Prx II action in NSCLC gefitinib-resistant cells has never been assessed using A549/GR cells.

Here, we examined the expression of Prxs and antioxidant enzymes in drug-resistant NSCLC and found that Prx II was highly expressed only in A549/GR cells, but not in A549 cells. This increase in expression was associated with increases in tumor cell growth and proliferation via activation of pJNK and suppression of apoptosis signaling in the A549/GR cells. Prx II may have an essential role in A549/GR cancer cell survival.

\section{MATERIALS AND METHODS}

\section{Establishment of EGFR-TKI-resistant cell lines and A549/GR-shPrx II cell lines}

The human NSCLC cell lines ${ }^{5}$ A549, H460, PC-9 and HCC827 were maintained in RPMI 1640 medium (Gibco, Grand Island, NY, USA) supplemented with $5 \%$ heat-inactivated fetal bovine serum (Hyclone, South Logan, UT, USA), 2 mm glutamine and antibiotics $\left(100 \mathrm{U} \mathrm{ml}^{-1}\right.$ penicillin and $100 \mu \mathrm{g} \mathrm{ml}^{-1}$ streptomycin) at $37^{\circ} \mathrm{C}$ in a humidified atmosphere of $5 \% \mathrm{CO}_{2}$. The A549 (GR, CR, PR, GemR and IR), ${ }^{5}$ PC-9 (GR, CR, ER, CLR and ZDR), H460 (CR, PR and IR) and HCC827 (GR, CR, PR, ER and CLR) cell lines have been established in previous studies (GR, gefitinib resistant; CR, cisplatin resistant; CLR, CL-387 and 785 resistant; PR, paclitaxel resistant; ZDR, ZD6474 resistant; ER, erlotinib resistant; GemR, gemcitabine resistant and IR, irradiation resistant). ${ }^{14}$ The A549/GR-shPrx II cell lines were established by the transfection of A549/GR cells with shPrx II-1, shPrx II-2, shPrx II-3 and scrambled short hairpin RNA. Then, G418 screening was performed as previously described. ${ }^{15}$

\section{Flow cytometry analyses}

The ROS in the A549, A549/GR and A549/GR-shPrx II cells were determined using $2^{\prime}, 7^{\prime}$-dichlorodihydrofluorescein diacetate (DCF-DA; Invitrogen, Grand lsland, NY, USA). Cells were incubated with $20 \mathrm{~mm}$ of DCF-DA for $15 \mathrm{~min}$ at $37^{\circ} \mathrm{C}$ and then washed with phosphate-buffered saline (PBS). Cell apoptosis was measured by washing the cells with PBS, staining them with Annexin V Binding Buffer (BD Biosciences, San Diego, CA, USA), and labeling them with Annexin V-FITC (BD Biosciences), as recommended by the manufacturer. Cell cycle analyses were performed as follows: the stimulated cells were washed once with PBS. Cell pellets were fixed with $70 \%$ ethanol in PBS, stained with propidium iodide-PE (PI, BD Biosciences) for $20 \mathrm{~min}$ on ice and analyzed with a FACSCalibur (Becton Dickinson, San Diego, CA, USA). ${ }^{16}$

\section{Soft agar colony assays}

Soft agar colony assays was performed as previously described. ${ }^{17}$ A549, A549/GR and A549/GR-shPrx II $\left(3 \times 10^{3}\right)$ were suspended in $1 \mathrm{ml}$ of Dulbecco's Modified Eagle's medium containing $0.3 \%$ agar in cell growth medium and plated in triplicate over the first layer of $0.6 \%$ agar in the cell growth medium. The six-well plates were incubated at $37^{\circ} \mathrm{C}$ and $5 \% \mathrm{CO}_{2}$ for 2 weeks. After macroscopically visible clones appeared, the culture was terminated; then, the cells were rinsed, fixed and subjected to Giemsa staining (Sigma-Aldrich, St Louis, MO, USA). The clones were counted, and the cloning efficiency was calculated according to the following formula: cloning efficiency $(\%)=$ number of clones/number of inoculations $\times 100 \% .{ }^{15}$

\section{Quantitative reverse transcriptase-PCR}

Quantitative reverse transcriptase-PCR was performed as previously described. ${ }^{16}$ RNA was isolated from the cell lines at each passage using TRIZOL (Invitrogen). Complementary DNA was synthesized from $1 \mathrm{mg}$ of total RNA using oligo dT18 primers and Superscript reverse transcriptase (Bioneer, Daejeon, Korea) in a final volume of $20 \mu \mathrm{l}$. For standard PCR, $1 \mu \mathrm{l}$ of first-strand complementary DNA was used as a template for PCR amplification with Taq DNA polymerase (Fermentas, Grand Island, NY, USA). Quantitative reverse transcriptase-PCR reactions were performed using SYBR Green JumpStart Taq ReadyMix (Takara, Shiga, Japan) with an Exicycler 96 Real-Time Quantitative Thermal Block (Bioneer). Relative quantification was achieved by normalization to endogenous $\beta$-actin. The primers used are shown in Table 1.

\section{Xenograft mice}

The tumorigenicities of the A549, A549/GR and A549/GR-shPrx II cells were assayed by the subcutaneous inoculation of $1 \times 10^{5}$ cells resuspended in a mixture of matrigel and PBS into the flanks of 5 -week-old athymic BALB/c female nude mice ( $n=5$ per group). Tumor size was measured using calipers (calculated volume $=$ shortest diameter ${ }^{2} \times$ longest diameter/2) at each 3-day interval. Five weeks after cell inoculation, the grafts were removed and photographed. ${ }^{15}$

\section{Western blot}

Western blot was performed as previously described. ${ }^{17}$ Protein samples were separated by $12 \%$ SDS-PAGE and transferred to nitrocellulose membranes. Blotting was carried out with rabbit or mouse antibodies against Prx II (Lab Frontier, Seoul, Korea), c-Raf (Cell Signaling Technology, Danvers, MA, USA), pMEK (Cell Signaling Technology), pERK (Cell Signaling Technology), ERK (Cell Signaling Technology), pJNK (Cell Signaling Technology), JNK (Cell Signaling Technology), BCL-2 (Cell Signaling Technology), caspase-3 (Cell Signaling Technology), cleaved caspase-3 (Cell Signaling Technology), poly ADP-ribose polymerase (Cell Signaling Technology) and cleaved poly ADP-ribose polymerase (Cell Signaling Technology).

\section{Data analysis}

Statistical analysis was performed using analysis of variance. A $P$-value of $<0.05$ was considered significant.

\section{RESULTS}

High expression of Prx II in A549/GR cells

To investigate the relevance of Prxs in drug-resistant non-small cell lung carcinoma, we assessed the expression of Prxs in several EGFR-TKI-resistant cells by western blotting. As shown in Figure 1a, Prx II expression was dramatically increased in the 
A549/GR cells, but not in the A549 cells resistant to other drugs. In the other drug-resistant lung carcinoma cells, all six Prxs were stably expressed. We determined the mRNA levels of

Table 1 Real-time PCR primers used to amplify antioxidant enzyme mRNAs

\begin{tabular}{|c|c|}
\hline Gene & Primer sequence \\
\hline \multicolumn{2}{|l|}{$\operatorname{Prx} 1$} \\
\hline Forward & 5'-GGGCACACAAAGGTGAAGTC-3' \\
\hline Reverse & 5'-GCTGTTATGCCAGATGGTCAG-3' \\
\hline \multicolumn{2}{|l|}{ Prx II } \\
\hline Forward & 5'-TCATCCACGTTGGGCTTAAT-3' \\
\hline Reverse & 5'-CCAGGCCTTCCAGTACACAG-3' \\
\hline \multicolumn{2}{|l|}{$\operatorname{Prx} I / I$} \\
\hline Forward & 5'-GACGCTCAAATGCTTGATGA-3' \\
\hline Reverse & 5'-GATTTCCCGAGACTACGGTG-3' \\
\hline \multicolumn{2}{|l|}{$\operatorname{Prx} I V$} \\
\hline Forward & 5'-TCCTTGTCTTCGAGGGGTAT-3' \\
\hline Reverse & 5'-TTGGCGACAGACTTGAAGAA-3' \\
\hline \multicolumn{2}{|l|}{$\operatorname{Prx} V$} \\
\hline Forward & 5'-TTCAAACACCTCCACTGCTG-3' \\
\hline Reverse & 5'-AGTGAAGGAGAGTGGGCGTC-3' \\
\hline \multicolumn{2}{|l|}{$\operatorname{Prx} V I$} \\
\hline Forward & 5'-CAAGCTCCCGATTCCTATCA-3' \\
\hline Reverse & 5'-GTTGAGGACCATCTTGCCTG-3' \\
\hline \multicolumn{2}{|l|}{$G P x-1$} \\
\hline Forward & 5'-AAGAGCATGAAGTTGGGCTC-3' \\
\hline Reverse & 5'-CAACCAGTTTGGGCATCAG-3' \\
\hline \multicolumn{2}{|l|}{$G P x-2$} \\
\hline Forward & 5'-TTTTGGACAAGGGTGAAGGT-3' \\
\hline Reverse & 5'-TGCAACCAATTTGGACATCA-3' \\
\hline \multicolumn{2}{|l|}{$G P x-3$} \\
\hline Forward & 5'-GGATGTACTCCTCCCCATCA-3' \\
\hline Reverse & 5'-GCCGGGGACAAGAGAAGT-3' \\
\hline \multicolumn{2}{|l|}{ SOD 1} \\
\hline Forward & 5'-CCACACCTTCACTGGTCCAT-3' \\
\hline Reverse & 5'-CTAGCGAGTTATGGCGACG-3' \\
\hline \multicolumn{2}{|l|}{ SOD 2} \\
\hline Forward & 5'-TGACCACCACCATTGAACTT-3' \\
\hline Reverse & 5'-CGTCACCGAGGAGAAGTACC-3' \\
\hline \multicolumn{2}{|l|}{ SOD 3} \\
\hline Forward & 5'-GAGCAGGCAGGAACACAGTA-3' \\
\hline Reverse & 5'-GGGTGCAGCTCTCTTTTCAG-3' \\
\hline
\end{tabular}

The PCR amplification proceeded as follows: 40 cycles of $94^{\circ} \mathrm{C}$ for $30 \mathrm{~s}, 60^{\circ} \mathrm{C}$ for $30 \mathrm{~s}$ and $72^{\circ} \mathrm{C}$ for $30 \mathrm{~s}$. antioxidant enzymes, including the Prxs in the A549/GR cells, and found that Prx II expression was increased by $\sim 600$-fold in the A549/GR cells compared with the A549 cells and that the expression levels of Prx IV, Prx VI and SOD 1 were also significantly increased (Figure 1b). Because it is well known that Prx II is not at all or only slightly expressed in the lung tumors of NSCLC patients, we hypothesized that methylation may be involved in the regulation of Prx II gene expression. Therefore, we examined methylation in the upstream region of the Prx II gene in Beas2B, A549 and A549/GR cells (Figures 1c and d). As expected, a 5'-CCGG-3' methylation site was found upstream of the Prx II gene that was highly methylated in the A549 cells, but not in the other cells. All these data suggest that gefitinib resistance may be involved in the demethylation of $\operatorname{Pr} x$ II genes, thereby increasing the expression of Prx II in the A549/GR cells.

\section{Prx II regulated JNK and apoptosis signaling in A549/GR cells}

We investigated whether the high expression of Prx II in A549/GR cells may be involved in the regulation of ROS, apoptosis and the cell cycle. First, we observed morphologic changes in the A549/GR cells compared with the A549 cells, including spindle-shaped cells with a loss of polarity and the increased formation of pseudopodia (Figure 2a). ${ }^{5}$ The percentages of DCF-DA and Annexin V-positive cells were significantly reduced in the A549/GR cells compared with the A549 cells. In addition, the A549/GR cells in G1 phase were significantly decreased compared with the A549 cells, and those at $\mathrm{G} 2 / \mathrm{M}$ underwent cell cycle arrest (Figures $2 \mathrm{~b}$ and c).

To confirm the relevance of Prx II in the regulation of ROS, apoptosis and the cell cycle in the A549/GR cells, we examined the effects of its downregulation. A549/GR cell lines stably transfected with shPrx II-1, shPrx II-2, shPrx II-3 (A549/GR-shPrx II) and short hairpin RNA-scrambled (control) were established by liposomal transfection and G418 screening. The knockdown efficiencies for the shPrx II-1, shPrx II-2 and shPrx II-3 groups were 75.4, 69.1 and $65.6 \%$, respectively. Western blot analysis showed the remarkably decreased expression of Prx II in all three shPrx II transfection groups (Figure 2d). We used A549/GR-shPrx II-1 for all experiments. The spindle shape and pseudopodia formation of the A549/GR-shPrx II cells were not altered. These cells grew slowly compared with the A549/GR cells (Figure 2e). We investigated the level of ROS and apoptosis in the A549/GR-shPrx II cells using flow cytometry. The percentages of DCF-DA and Annexin V-positive cells were significantly increased in the A549/GR-shPrx II cells compared with the A549/GR cells (Figure 2f). The cell cycle distributions in the transfection groups were then determined. The percentage of G1 cells was significantly increased by the downregulation of $\operatorname{Prx}$ II in the A549/GR cells, and the number of cells in G2/M was significantly decreased in the A549/GR-shPrx II cells compared with the A549/GR cells (Figure $2 \mathrm{~g}$ ). To understand the underlying mechanism of Prx II in the regulation of apoptosis and the cell cycle, we examined 
A
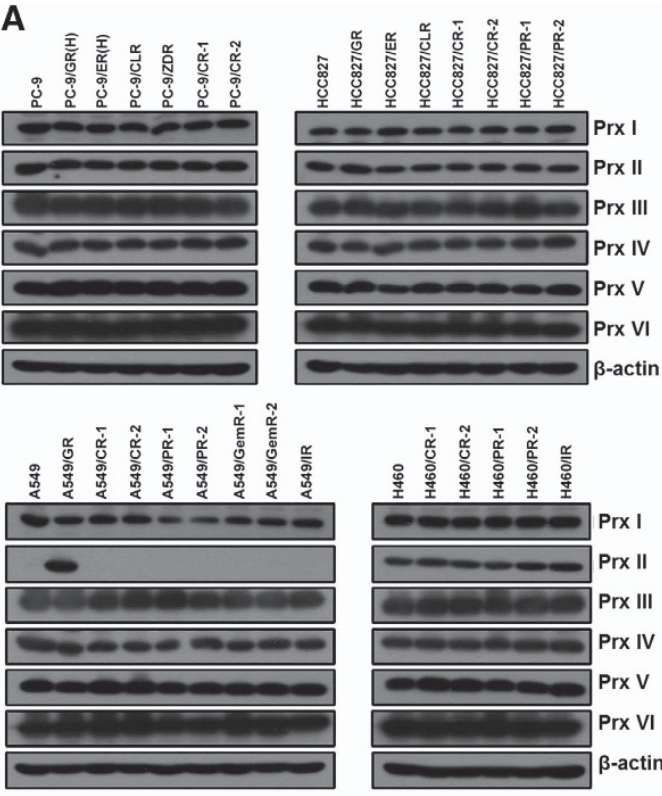

C
B
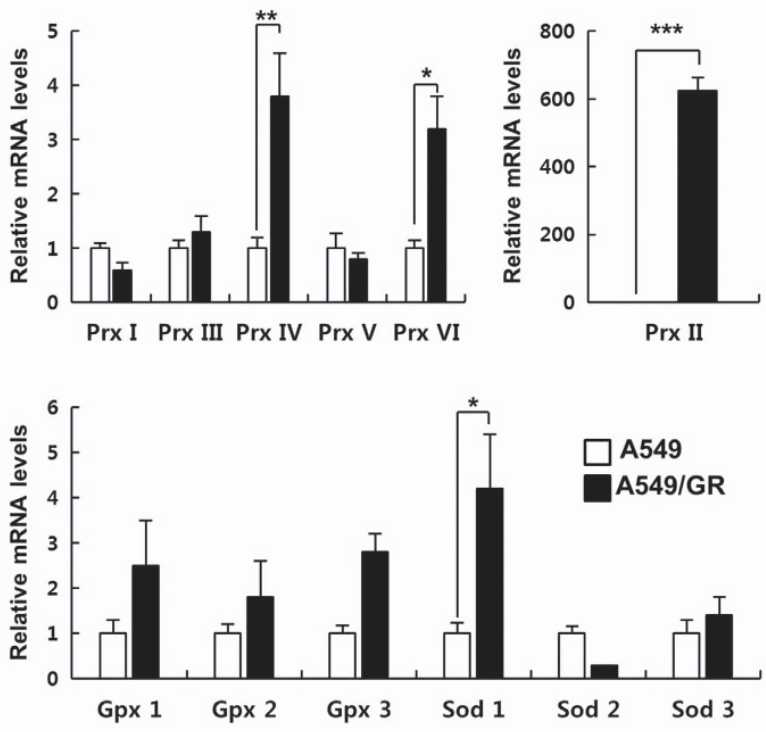

D
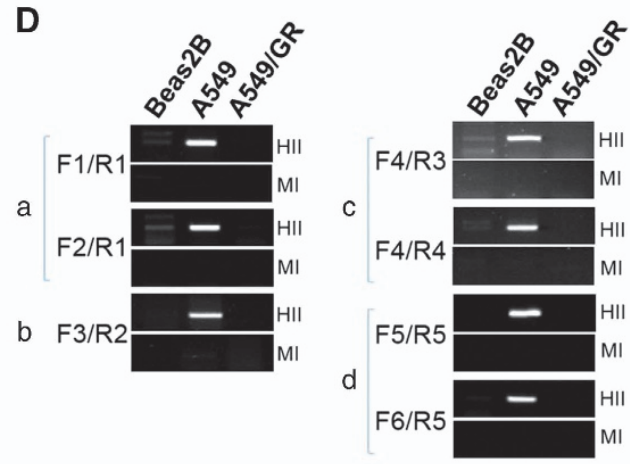

Figure 1 Expression of antioxidant enzymes and DNA methylation state at peroxiredoxin II (Prx II) promoter in NSCLC cells. (A) Western blot analysis of Prxs in A549 gefitinib- and EGFR-TKI-resistant cells. (B) Quantitative reverse transcriptase-PCR analysis of Prxs and other antioxidant enzymes in A549/GR cells. (C) Sequence information of the Prx II proximal promoter region used for DNA methylation analysis. The Hpall/Mspl recognition sequence ( $5^{\prime}-\mathrm{CCGG}-3^{\prime}$ ) is italicized and boxed (a, b-1, b-2, c and d). The forward primers (F1-F6) are underlined, and the reverse primers (R1-R6) are italicized. Transcription start site, +1. (D) DNA methylation analysis using restriction enzyme digestion. Genomic DNA was extracted from the indicated cell lines and digested with either Hpall (HII) or Mspl (MI). Both enzymes recognize 5'-CCGG-3', but Hpall is unable to cut DNA when the internal cytosine is methylated. The combinations of primers used in amplifying each of the Hpall/Mspl sites (a-d) are indicated. The data are the mean \pm s.e.m. $(n=16) * P<0.05, * * P<0.01$ and $* * * P<0.001$.

the expression of signaling molecules associated with cell proliferation and apoptosis by western blotting. MAPK/ERK pathway activity was remarkably reduced in the A549/GR cells regardless of the downregulation of Prx II compared with the A549 cells (Figure 2h). However, pJNK expression was increased in the A549/GR cells and was significantly decreased in the A549/GR-shPrx II cells. Next, to investigate whether Prx II may be involved in the regulation of mitochondrial antiapoptosis proteins in the A549/GR cells, we examined the expression of $\mathrm{Bcl}-2$ and apoptosis signaling molecules. The downregulation of Prx II led to the decreased expression of $\mathrm{Bcl}-2$ and the increased expression of cleaved caspase- 3 and poly ADP-ribose polymerase cleavage in the A549/GR cells
(Figure 2i). All these findings suggest the involvement of Prx II in the activation of JNK and apoptotic signaling molecules in A549/GR cells.

\section{Effects of Prx II downregulation on cell proliferation and tumor growth}

To investigate whether Prx II downregulation affects cell proliferation and tumor growth, these processes were examined with a colony formation assay and in nude mice using A549, A549/GR and A549/GR-shPrx II cells. As shown in Figure 3a, colony numbers were increased in the A549/GR cells and were significantly diminished in the A549/GR-shPrx II cells. In addition, the nude mice experiments revealed significantly 
a

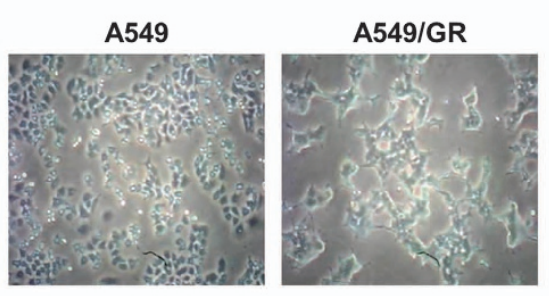

C

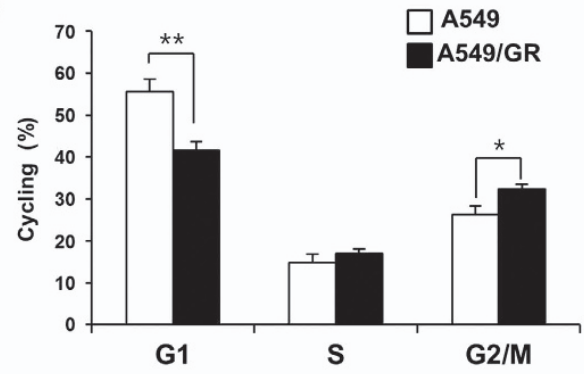

b

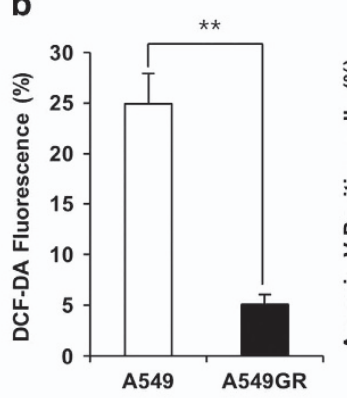

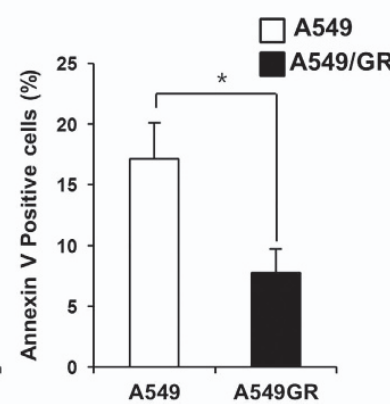

d

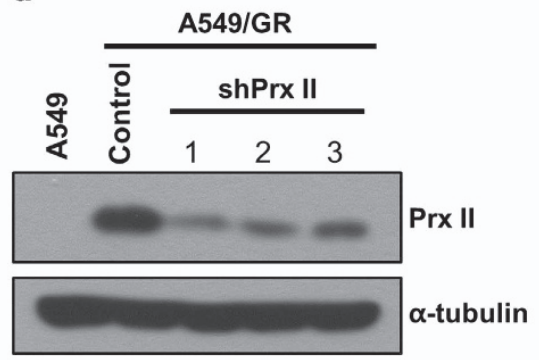

e
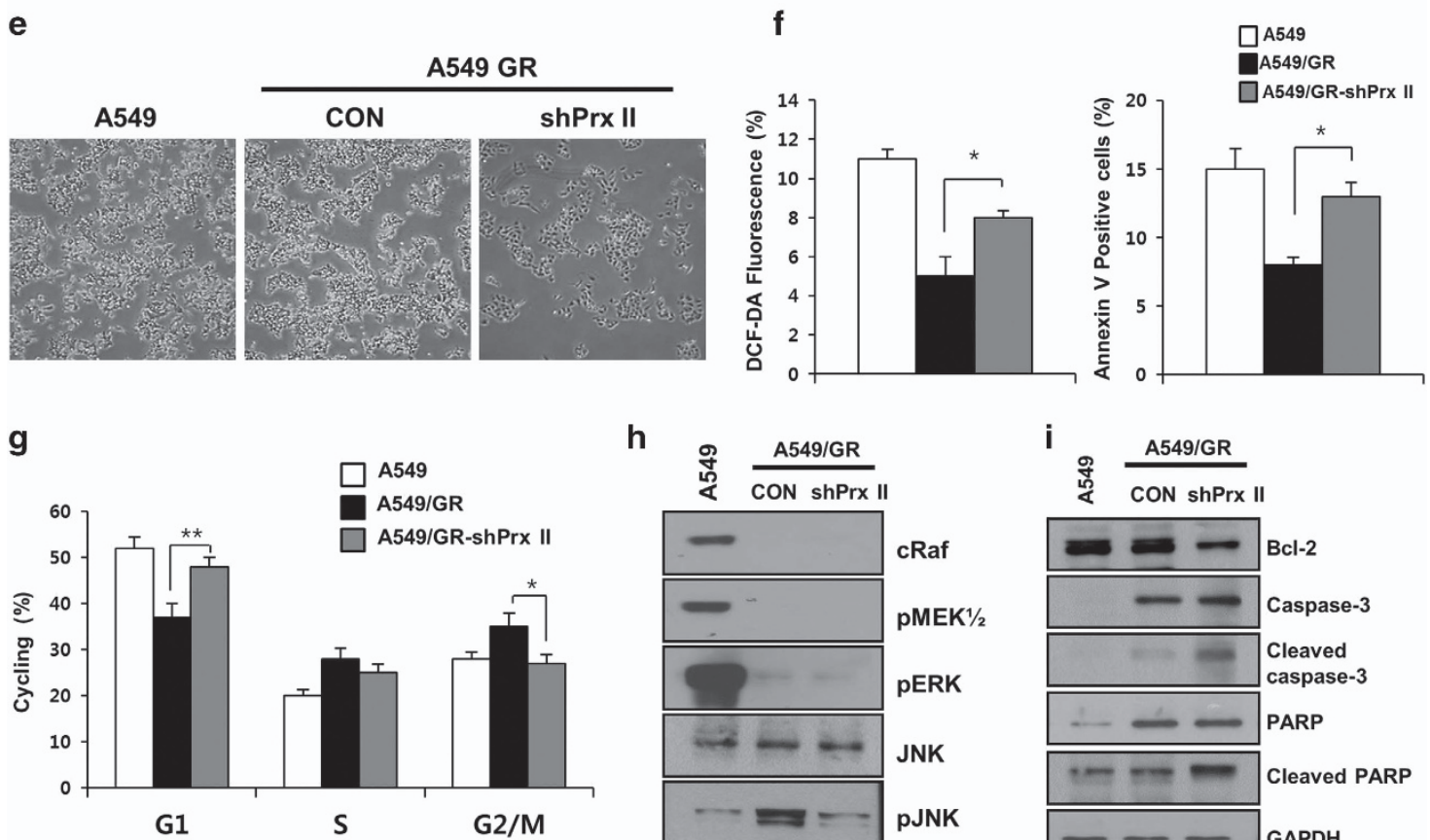

h
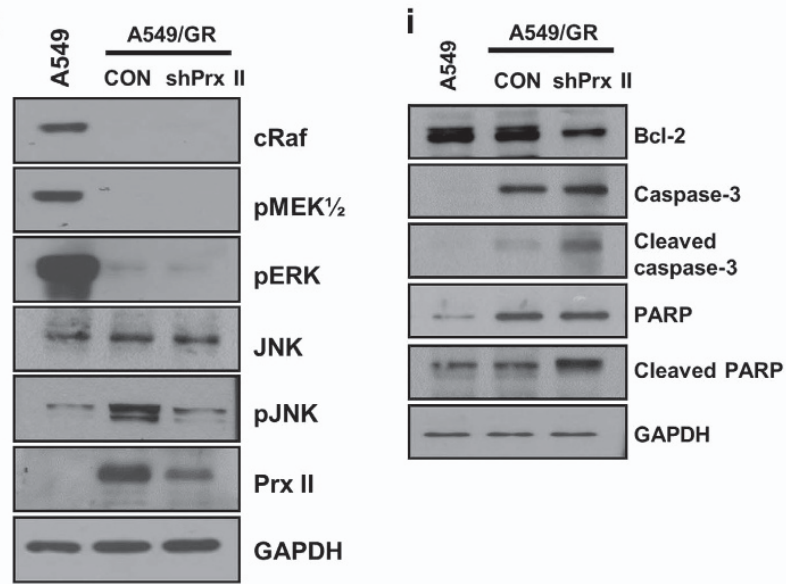

Figure 2 Peroxiredoxin II (Prx II) activates JNK signaling and suppresses apoptosis in A549/GR cells. (a) Microscopic observations of A549 and A549/GR cells. (b) Reactive oxygen species (ROS) and apoptosis levels in A549 and A549/GR cells. (c) Percentages of A549 and A549/GR cells in the G1, S and G2/M stages are shown. (d) The Prx II protein levels were significantly reduced in the three A549/GR-shPrx II cell lines compared with the A549/GR cells transfected with scrambled short hairpin RNA (control). (e) Microscopic observations of the A549/GR-shPrx II cells. (f) ROS and apoptosis levels in the A549/GR and A549/GR-shPrx II cells. (g) Percentages of A549, A549/GR and A549/GR-shPrx II cells at the G1, S and G2/M stages are shown. (h) Lysates from the A549, A549/GR (CON) and A549/GR-shPrx II cells were subjected to western blotting. The indicated antibodies were used to assess the levels of ERK/MAPK and JNK pathway marker proteins. (i) The expression levels of cleaved caspase-3 and cleaved poly ADP-ribose polymerase (PARP) in the A549, A549/GR (CON) and A549/GR-shPrx II cells were determined by western blot. GAPDH was used as an internal control. The data are the mean \pm s.e.m. $(n=6){ }^{*} P<0.05$ and ${ }^{*} P<0.01$. 
a
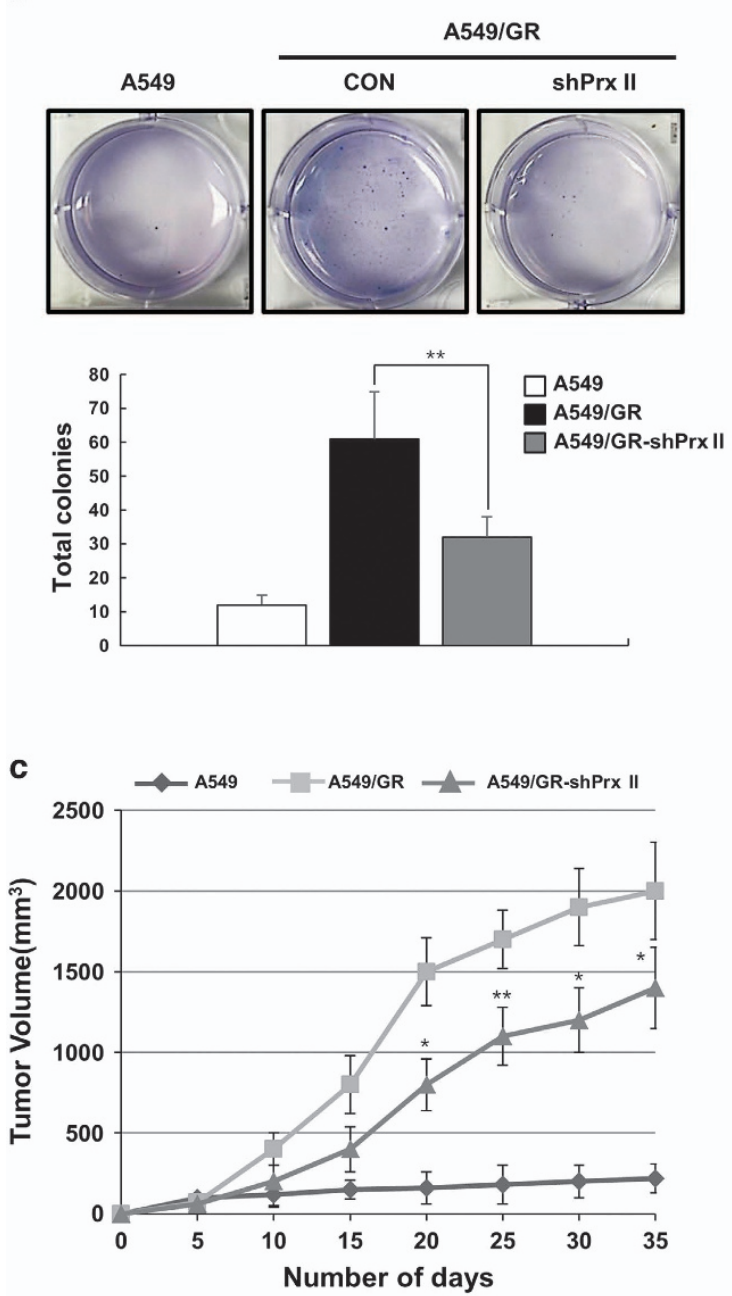

b
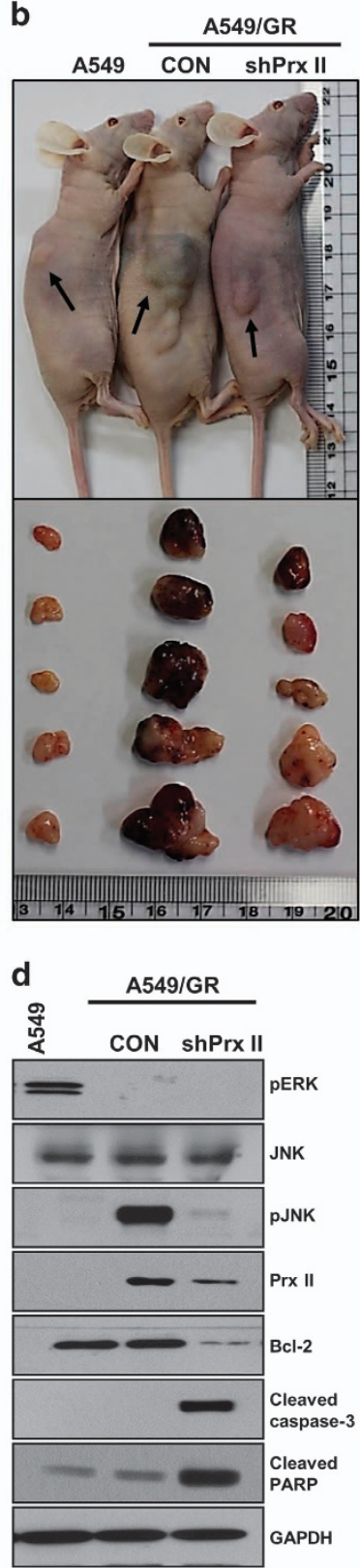

Figure 3 Peroxiredoxin II (Prx II) expression is essential for tumor growth of A549/GR cells in xenograft model. (a) Soft agar assays were performed with A549, A549/GR (CON) and A549/GR-shPrx II cells. (b) Representative image of tumor formation in nude mice. (c) Tumor volumes were observed for 35 days after the injection of A549 ( $>$ ), A549/GR (CON; $\square$ ) and A549/GR-shPrx II ( $\Delta$ ) cells into nude mice. (d) Western blot analysis of pJNK pathways and apoptosis signal markers in A549, A549/GR (CON) and A549/GR-shPrx II tumor tissues. GAPDH was used as an internal control. The data are the mean \pm s.e.m. $(n=5) * P<0.05$ and $* * P<0.01$.

decreased tumor growth in the mice treated with the A549/GR-shPrx II cells compared with the A549/GR cells (Figures $3 \mathrm{~b}$ and $\mathrm{c}$ ). These data indicate that Prx II may be essential for tumor cell growth in A549/GR cells.

To confirm whether Prx II downregulation affects the expression of pJNK and apoptosis-related signaling molecules, we performed western blotting to evaluate tumor tissues from nude mice. As shown in Figure 3d, pJNK expression was greatly increased in the A549/GR cells and was surprisingly decreased in the A549/GR-shPrx II cells. Additionally, the expression levels of apoptosis-related signaling molecules were also significantly regulated, as shown in Figure 2i. Bcl-2 expression was significantly decreased and the expression of cleaved caspase- 3 and cleaved poly ADP-ribose polymerase were remarkably increased in the A549/GR-shPrx II cells compared with the control A549/GR cells (Figure 3d). These results strongly suggest that Prx II has an important role in tumor proliferation through the activation of JNK and the downregulation of the apoptosis signaling pathway in A549/GR cells. 


\section{DISCUSSION}

We have previously reported the expression of Prx isotypes in human adenocarcinoma samples and numerous lung cell lines, including adenocarcinoma and non-transformed cells. The protein levels of Prx III, V and VI were unaltered between the normal and tumor tissues, whereas Prx II was greatly decreased in the tumor tissues compared with the normal tissues. ${ }^{12}$ However, the augmented expression of Prx II has been reported in a variety of distinct types of human cancers, including HCC. ${ }^{18}$ In addition, Prx II has been linked to cell proliferation, apoptosis and angiogenesis in tumors. ${ }^{19}$ These data suggest that this enzyme may also function in lung cancer cells. Therefore, we hypothesized that it may be involved in cancer cell survival, increasing in expression in malignant cancer cells, especially those that are drug resistant. In this study, we demonstrated that Prx II may have an essential role in A549/GR cancer cell survival. The Prx II mRNA level was increased by $\sim 600$-fold in the A549/GR cells compared with the A549 cells. Differences in the expression of Prx II between the A549 and A549/GR cells were associated with the methylation status of the upstream region of the Prx II gene. High expression of Prx II resulted in the downregulation of ROS and cell death and the upregulation of cell cycle progression in the A549/GR cells. Further, the activation of JNK was prominently observed in association with tumor proliferation following induction by Prx II in the nude mice. These results suggest that Prx II has an important role in cancer cell survival through modulating apoptosis and pJNK signaling molecules by promoting the downregulation of ROS levels in A549/GR cells.

MAPK proteins have key roles in cellular events, affecting diverse end points, including cell proliferation, differentiation, survival and death. ${ }^{20}$ ERK is activated in response to growth stimuli in cancer, and its indirect and direct targeting have both been shown to cause tumor cell death. ${ }^{20}$ However, the phosphorylation of ERK was decreased in the A549GR cells compared with the A549 cells. On the other hand, pJNK was strongly induced in the A549GR cells showing high Prx II expression. JNK, which is referred to as stress-activated protein kinase, is activated by various stress stimuli, such as DNA damage caused by oxidative stress. ${ }^{21}$ The Prx II-dependent regulation of the JNK pathway is essential for the completion, rather than the initiation, of DNA repair. Previous studies have suggested that Prx II is a novel anti-cancer target that regulates DNA repair in cancer cells and protects against DNA-damaging agents in HeLa cells. ${ }^{21}$ The biological function of Prx II in relation to tumorigenesis is somewhat complex. Prx II sensitizes cancer cells to chemotherapeutic agents by impairing JNK-dependent DNA repair. ${ }^{21}$ Overall, the present study characterized the role of ROS-induced alterations in the MAPK signaling pathway, especially the activation of JNK, as a responsible mediator of Prx II-induced apoptosis. The high expression of Prx II contributed to JNK activation by reducing the ROS levels in the A549GR cells. The Prx II-dependent regulation of the JNK pathway is essential for the completion of DNA repair in HeLa cells. ${ }^{21}$ ROS protect against Prx II-mediated antioxidative surveillance, thereby activating JNK in phospholamban-deficient and protein phosphatase 1 inhibitor 1-overexpressed mice. ${ }^{22}$ Prx II significantly protects A549GR cells from $\mathrm{H}_{2} \mathrm{O}_{2}$-induced apoptosis and necrosis, while downregulating oxidative stress. In the present study, we found that Prx II was involved in the activation of JNK and apoptotic signaling molecules in the A549/GR cells. The downregulation of Prx II also reduced the expression of antiapoptotic proteins, such as Bcl-2. Gefitinib causes a rapid increase in the level of $\mathrm{Bcl}-2$ through transcriptional and posttranslational mechanisms and inhibits tumor cell growth in human NSCLCs. ${ }^{6}$ Therefore, it is likely that the ROS/JNK pathway, which is associated with signaling, has an important role in Prx II-induced apoptosis in A549/GR cells. We are currently attempting to elucidate the direct involvement of the Prx-mediated surveillance of ROS in the regulation of the A549/GR stemness genes by JNK. Recently, the acquisition of an epithelial-mesenchymal transition, a feature highly reminiscent of cancer stem cells in the A549/GR cell line, has been demonstrated. ${ }^{15}$ Furthermore, based on these results, in the future, we will focus on identifying the functional role of Prx II in the maintenance of cancer stem cells in the A549/GR cell line.

In conclusion, our data provide evidence that Prx II expression has a functional role in the maintenance of JNK signaling in A549/GR cells.

\section{CONFLICT OF INTEREST}

The authors declare no conflict of interest.

\section{ACKNOWLEDGEMENTS}

This work was supported by the Mid-career Researcher Program through an NFR grant funded by the MSIP (OGM0021312) the World Class Institute (WCI) Program of the National Research Foundation of Korea (NRF) MSIP NRF (WCI 2009-002); the National Research Foundation of Korea (2010-0020877), funded by the Korean government; and the KRIBB Research Initiative Program (KGM3141312) of Korea. D-YY was partially supported by the Priority Research Centers Program (2012-0006686).

1 Jemal A, Siegel R, Ward E, Murray T, Xu J, Smigal C et al. Cancer statistics, 2006. CA Cancer J Clin 2006; 56: 106-130.

2 Lam WK, Watkins DN. Lung cancer: future directions. Respirology 2007; 12: 471-477.

3 Mehta AA, Jose WM, Pavithran K, Triavadi GS. The role of gefitinib in patients with non-small-cell lung cancer in India. Indian J Palliat Care 2013; 19: 48-53.

4 Paez JG, Janne PA, Lee JC, Tracy S, Greulich H, Gabriel S et al. EGFR mutations in lung cancer: correlation with clinical response to gefitinib therapy. Science 2004; 304: 1497-1500.

5 Rho JK, Choi YJ, Lee JK, Ryoo BY, Na II, Yang SH et al. Epithelial to mesenchymal transition derived from repeated exposure to gefitinib determines the sensitivity to EGFR inhibitors in A549, a non-small cell lung cancer cell line. Lung Cancer 2009; 63: 219-226.

6 Wu J, Min R, Wu M, Chen W. Gefitinib induces mitochondrial-dependent apoptosis in Saccharomyces cerevisiae. Mol Med Rep 2011; 4: 357-362.

7 Moller P, Wallin H.. Adduct formation, mutagenesis and nucleotide excision repair of DNA damage produced by reactive oxygen species and lipid peroxidation product. Mutat Res 1998; 410: 271-290.

8 Kowaltowski AJ, Netto LE, Vercesi AE. The thiol-specific antioxidant enzyme prevents mitochondrial permeability transition. Evidence for the 
participation of reactive oxygen species in this mechanism. J Biol Chem 1998; 273: 12766-12769.

9 Li S, Peterson NA, Kim MY, Kim CY, Hung LW, Yu M et al. Crystal Structure of AhpE from Mycobacterium tuberculosis, a 1-Cys peroxiredoxin. J Mol Biol 2005; 346: 1035-1046.

10 Jang HH, Lee KO, Chi YH, Jung BG, Park SK, Park JH et al. Two enzymes in one; two yeast peroxiredoxins display oxidative stress-dependent switching from a peroxidase to a molecular chaperone function. Cell 2004; 117: 625-635.

11 Zhang B, Wang Y, Su Y. Peroxiredoxins, a novel target in cancer radiotherapy. Cancer Lett 2009; 286: 154-160.

12 Park YH, Kim SU, Lee BK, Kim HS, Song IS, Shin HJ et al. Prx I suppresses K-ras-driven lung tumorigenesis by opposing redox-sensitive ERK/cyclin D1 pathway. Antioxid Redox Signal 2013; 19: 482-496.

13 Lehtonen ST, Svensk AM, Soini Y, Paakko P, Hirvikoski P, Kang SW et al. Peroxiredoxins, a novel protein family in lung cancer. Int J Cancer 2004; 111: 514-521.

14 Chang Y, Kim SY, Choi YJ, So KS, Rho JK, Kim WS et al. Neuroendocrine differentiation in acquired resistance to epidermal growth factor receptor tyrosine kinase inhibitor. Tuberc Respir Dis (Seoul) 2013; 75: 95-103.

15 Jung MJ, Rho JK, Kim YM, Jung JE, Jin YB, Ko YG et al. Upregulation of CXCR4 is functionally crucial for maintenance of stemness in drug-resistant non-small cell lung cancer cells. Oncogene 2013; 32: 209-221.

16 Kwon TH, Han YH, Hong SG, Lee DJ, Ha HL, Kang SW et al. Reactive oxygen species mediated DNA damage is essential for abnormal erythropoiesis in peroxiredoxin II (-/-) mice. Biochem Biophys Res Commun 2012; 424: $189-195$

17 Kim JH, Shin HJ, Ha HL, Park YH, Kwon TH, Jung MR et al. Methylsulfonylmethane suppresses hepatic tumor development through activation of apoptosis. World J Hepatol 2014; 6: 98-106.
18 Diao S, Zhang JF, Wang H, He ML, Lin MC, Chen $\mathrm{Y}$ et al. Proteomic identification of microRNA-122a target proteins in hepatocellular carcinoma. Proteomics 2010; 10: 3723-3731.

19 Kang DH, Lee DJ, Lee KW, Park YS, Lee JY, Lee SH et al. Peroxiredoxin II is an essential antioxidant enzyme that prevents the oxidative inactivation of VEGF receptor-2 in vascular endothelial cells. Mol Cell 2011; 44: 545-558.

20 Xia Z, Dickens M, Raingeaud J, Davis RJ, Greenberg ME. Opposing effects of ERK and JNK-p38 MAP kinases on apoptosis. Science 1995; 270 . $1326-1331$.

21 Lee KW, Lee DJ, Lee JY, Kang DH, Kwon J, Kang SW.. Peroxiredoxin II restrains DNA damage-induced death in cancer cells by positively regulating JNK-dependent DNA repair. J Biol Chem 2011; 286: 8394-8404.

22 Zhao W, Fan GC, Zhang ZG, Bandyopadhyay A, Zhou X, Kranias EG. Protection of peroxiredoxin II on oxidative stress-induced cardiomyocyte death and apoptosis. Basic Res Cardiol 2009; 104: 377-389.

(c) (i) $\odot$ This work is licensed under a Creative Commons Attribution-NonCommercial-NoDerivs

International License. The images or other third party material in this article are included in the article's Creative Commons license, unless indicated otherwise in the credit line; if the material is not included under the Creative Commons license, users will need to obtain permission from the license holder to reproduce the material. To view a copy of this license, visit http://creativecommons.org/licenses/by-nc-nd/4.0/ 